LA-UR- $10-0359$

Approved for public release; distribution is unlimited.
Title: Recent Advances in Nuclear Fission Theory: Pre- and PostScission Physics

Author(s): P.Talou, T.Kawano, O.Bouland, J. E. Lynn, P.Moller, M.B.Chadwick

Intended for:
Proceedings of the International Conference on Nuclear Data for Science \& Technology ND2010 Jeju Island, Korea

April 26-30, 2010

Los Alamos National Laboratory, an affirmative action/equal opportunity employer, is operated by the Los Alamos National Security, LLC for the National Nuclear Security Administration of the U.S. Department of Energy under contract DE-AC52-06NA25396. By acceptance of this article, the publisher recognizes that the U.S. Government retains a nonexclusive, royalty-free license to publish or reproduce the published form of this contribution, or to aliow others to do so, for U.S. Government purposes. Los Alamos National Laboratory requests that the publisher identify this article as work performed under the auspices of the U.S. Department of Energy. Los Aiamos National Laboratory strongly supports academic freedom and a researcher's right to publish; as an institution, however, the Laboratory does not endorse the viewpoint of a publication or guarantee its technical correctness. 
Title: Recent Advances in Nuclear Fission Theory: Pre- and Post- Scission Physics Authors: P.Talou, T.Kawano, O.Bouland, J.E.Lynn, P.Moller, and M.B.Chadwick What: Proceedings of the International Conference on Nuclear Data for Science \& Technology ND2010

Where: Jeju Island, Korea

When: April 26-30, 2010

\section{Abstract:}

Recent advances in the modeling of the nuclear fission process for data evaluation purposes are reviewed. In particular, it is stressed that a more comprehensive approach to fission data is needed if predictive capability is to be achieved. The link between pre- and post-scission data is clarified, and a path forward to evaluate those data in a consistent and comprehensive manner is presented. Two examples are given: (i) the modeling of fission cross-sections in the R-matrix formalism, for which results for $\mathrm{Pu}$ isotopes from 239 to 242 are presented; (ii) the modeling of prompt fission neutrons in the Monte Carlo Hauser-Feshbach framework. Results for neutron-induced fission on $\mathbb{\$}^{\wedge}\{235\} \mathbb{W}$ are discussed. 


\title{
RECENT ADVANCES IN NUCLEAR FISSION THEORY: PRE- AND POST-SCISSION PHYSICS
}

\author{
P. Talou', a , T. Kawano', O. Bouland ${ }^{1,2}$, J.E. Lynn', P. Möller' ${ }^{1}$, and M.B. Chadwick ${ }^{3}$ \\ Theoretical Division, Los Alamos National Laboratory, Los Alamos, NM 87545, USA \\ ${ }^{1} \mathrm{CEA}$ DEN/LEPh, Cadarache, France \\ ${ }^{2} \mathrm{X}-\mathrm{CP}$, Computational Physics, Los Alamos National Laboratory, Los Alamos, NM 87545, USA \\ *Corresponding author. E-mail : \\ Received \\ Accepted for Publication
}

Recent advances in the modeling of the nuclear fission process for data evaluation purposes are reviewed. In particular, it is stressed that a more comprehensive approach to fission data is needed if predictive capability is to be achieved. The link between pre- and post-scission data is clarified, and a path forward to evaluate those data in a consistent and comprehensive manner is presented. Two examples are given: (i) the modeling of fission cross-sections in the R-matrix formalism, for which results for $\mathrm{Pu}$ isotopes from 239 to 242 are presented; (ii) the modeling of prompt fission neutrons in the Monte Carlo Hauser-Feshbach framework. Results for neutron-induced fission on ${ }^{235} \mathrm{U}$ are discussed.

KEYWORDS : Fission Cross-Section; Prompt Fission Neutrons and Gamma Rays; Monte Carlo Hauser-Feshbach

\section{MOTIVATION}

By any measure, understanding the nuclear fission process remains a daunting task even to this day. This peculiar collective behavior of some heavy nuclei is a hard challenge for standard quantum mechanics due to the sheer size of the number of degrees of freedom involved, and to the remaining mysteries clouding the nucleon-nucleon forces. Due to its intrinsic collective nature, however, only a few degrees of freedom appear critical. Niels Bohr realized that very early on after the experimental discovery of fission, and was able to describe correctly, albeit qualitatively only, the main features of this process. This remarkable achievement is illustrated in his seminal paper [1] published.. in 1939! Since this early period, much progress has been made in the interpretation of the fission process and in the prediction of many related data, such as fission cross sections, fission fragment yield distributions, fission fragment angular distributions, etc. Despite these undisputed progresses, modern evaluations of nuclear fission data still rely heavily on experimental data, as fission theory and modeling capabilities remain at the qualitative level for the most part. In addition, comprehensive studies allowing the prediction of many related data in a consistent manner remain elusive. In this presentation, we make the case for the development of such comprehensive studies, and present two recent modeling efforts that represent two pieces of this complicated puzzle. In a first part, the modeling of fission cross sections using the Bjørnholm-Lynn approach, based

\footnotetext{
a Corresponding author, e-mail: talou@lanl.gov
}

on R-matrix theory applied to the deformation channel, is presented. Implemented in modern statistical nuclear reaction codes, this approach can lead to significant advances toward the prediction, as opposed to adjustment, of fission cross sections. In a second part, an advanced modeling of the emission of prompt fission neutrons and gamma rays through Monte Carlo Hauser-Feshbach theory is described. The advantages of this approach compared to the existing models used in current evaluations are discussed. This paper concludes by addressing what should/could constitute a modern evaluation tool aimed at describing fission data in all their complexity.

\section{FISSION CROSS SECTION MODELING}

Obviously, an accurate modeling of fission cross sections is of great importance for many nuclear technologies. More recently, a renewed interest in this topic has emerged with the resurgence of advanced nuclear reactors and fuels, which involve fast neutron spectra and innovative fuel compositions. Indeed, the next generation of reactors would have to produce (much) more electricity than conventional reactors from the same amount of uranium ore, reduce significantly the amount of nuclear waste produced during the normal operation of the reactors, while maintaining the highest level of safety and security. Accurate fission cross sections for many nuclei, not all observed experimentally, are needed, and only advances in theory and modeling can tackle this challenge. 


\subsection{Hauser-Feshbach Theory}

In this work, we are only interested in modeling fission cross sections above the resolved resonance region, where the statistical Hauser-Feshbach equations can be applied. In the unresolved resonance region, well-known width fluctuation corrections have to be applied to the standard HauserFeshbach theory, and are taken into account in this work. In the Hauser-Feshbach theory, the cross-section for the reaction $(a, b)$ can be written as

$$
\sigma_{a b}^{J \pi}=\pi \lambda_{a}^{2} g(J) T_{a}^{J \pi} \frac{T_{b}}{\sum_{c} T_{c}} S_{a b}
$$

where $g(J)$ represents the spin statistical factor, and $T_{c}$ are the transmission coefficients for a particular reaction channel $c$. This equation assumes that the system first forms a compound nucleus characterized by the quantum numbers $J^{\pi}$, before decaying in the outgoing channel $b$. In the case of fission, a similar equation can be written where $T_{b}$ is replaced by a fission transmission coefficient $T_{f}$. The coefficient $S_{a b}$ is a correction factor that takes into account fluctuations from resonance to resonance. It becomes important when only few channels are open, and tends to increase the elastic cross-section at the expense of competing channels.

\subsection{Bjørnholm-Lynn Model}

In all modern nuclear reaction codes, the determination of $T_{f}$ is treated as a tunneling problem through onedimensional fission barriers. The characteristics of these barriers are often obtained empirically from various experimental cross-section data sets, or more recently, from microscopic [2] or macroscopic-microscopic [3] calculations. For many actinides, this one-dimensional fission path is characterized by a double-hump along the quadrupole elongation coordinate, due to microscopic corrections on top of a macroscopic liquid-drop term. The presence of this double-hump leads to intermediate structures in fission probabilities.

In the Bjørnholm-Lynn model [4], the R-matrix formalism is applied in the elongation channel that leads to fission. In the case of a double-humped fission barrier, this formalism calls for the distinction between class-I and classIl states, which lie respectively in the first (normally deformed) and second (super-deformed) wells. The minimum energy in the second well is generally $2-3 \mathrm{MeV}$ above the ground-state well. Hence the mean spacing $D_{l}$ of levels in the first well is significantly smaller than the mean spacing $D_{H}$ of class-II states.

In its simplest formulation, i.e., statistical limit, for which the coupling between class-I and class-II states is neglected, $T_{f}$ is calculated as

$$
T_{f}=\frac{T_{A} T_{B}}{T_{A}+T_{B}}
$$

where $T_{A, B}$ are individual transmission coefficients corresponding to the inner and outer fission barriers, respectively.
Those can be obtained by simple integration of the curve between the potential energy surface and the excitation energy at which $T_{f}$ is calculated.

The coupling between class-I and class-II states leads to a reformulation of Eq. 2. A given class-II state $\lambda_{1 /}$ can either decay to lower class-II states by electromagnetic radiation, decay to a normally deformed class-[ state, or couple to a state in the continuum by tunneling through the outer fission barrier. The coupling width of the state $\lambda_{11}$ to class-I states $\lambda_{1}$ is given by

$$
\Gamma_{\lambda_{11}(c)}=2 \pi\left\langle H_{c}^{2}\left(\lambda_{1 /} \lambda_{l}\right)\right\rangle / D_{l},
$$

where $H_{c}\left(\lambda_{l /} \lambda_{l}\right)$ is the coupling Hamiltonian. The average coupling width is related to the transmission coefficient across the inner barrier by

$$
2 \pi\left\langle\Gamma_{\lambda_{H}(c)}\right\rangle / D_{H}=T_{A} .
$$

Similarly, the average class-II fission width is related to the transmission coefficient $T_{B}$ across the outer barrier by

$$
2 \pi\left\langle\Gamma_{\lambda /(f)}\right\rangle / D_{I I}=T_{B}
$$

In the present work, we neglect the class-II radiation width, which would lead to delayed fission events.

At sub-barrier energies, assuming that both class-I and class-II states follow the uniform picket-fence model (no fluctuation of resonance spacings and widths), and averaging over the intermediate resonance structure, the average neutron-induced fission cross-section is rewritten as

$$
\left\langle\sigma_{n, f}^{J, \pi}\right\rangle=\pi \lambda^{2} g(J) \frac{T_{a}}{\left\{1+R^{2}+2 R \operatorname{coth}\left[\pi \Gamma_{\lambda_{1 /}} / D_{I I}\right]\right\}^{1 / 2}},
$$

with

$$
R=\Gamma_{\lambda / I} \Gamma_{l} D_{I I} /\left(\Gamma_{\lambda_{1 / n}} \Gamma_{\lambda_{l /(c)}} D_{l}\right)
$$

where $\Gamma_{I}$ is the sum of radiation and particle emission widths for class-I states. Taking into account resonance to resonance fluctuations (spacings, widths) will again modify this simplified formula. It is impossible to treat those fluctuations in a closed-form. Instead, we rely on the Monte Carlo technique to infer their impact on the average fission crosssections. Several approximations can be made depending on the excitation energy considered: sub-barrier, near barrier, or above barrier energies. More details about this procedure can be found in Ref. [5].

\subsection{Application to $\mathrm{Pu}$ Isotopes}

This formalism was applied to a suite of $\mathrm{Pu}$ isotopes, from 239 to 242 . In this preliminary work, fission cross sections were computed below the threshold for second-chance fission only. The preliminary results of these calculations are shown in Figure 1.

Many model input parameters enter in the formalism described above, but most of them can be inferred or/and constrained by experimental data. The level densities at the 


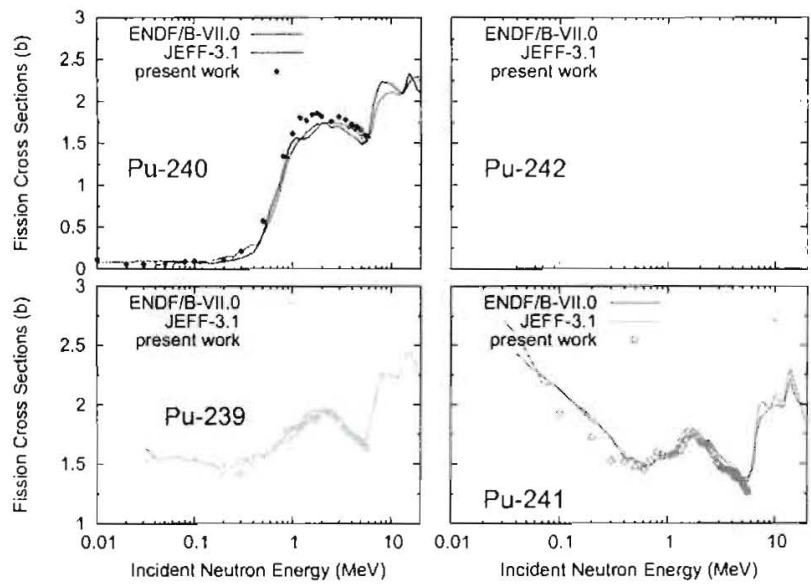

Fig. 1. Neutron-induced fission cross-sections calculated with the Monte Carlo technique (see text for details).

ground-state deformation, as well as the inner and outer barriers, play a crucial role in fitting observed cross-sections properly. In the present calculations, the level densities were represented as different phases of constant-temperature followed by a Fermi gas representation at higher energies. In some cases however, a combinatorial method was used, starting from single-particle states obtained in the FRLDM approach [3]. Fission barrier parameters, aside from level densities, were obtained from transfer reactions such as $(t, p f)$, which provide fission probabilities.

The role of intermediate structures in fission crosssection calculations is best seen on Fig. 2 where various assumptions for calculating ${ }^{239} \mathrm{Pu}(n, f)$ cross sections are compared. The impact of fluctuations of class-l and classII states is obviously more important at low-energies, while all calculations tend toward the simpler Hauser-Feshbach result with increasing excitation energy. Interestingly, the full Monte Carlo approach leads to an additional 20\% decrease in the fission cross-section in the 10-300 keV region, compared to the uniformed picket-fence model result (Eq. 6).

These calculations are preliminary, and several improvements are foreseen in the near future. The present results were obtained with a modern version of the AVXSF code written by J.E. Lynn. However, modern treatments of the radiative capture and of elastic and inelastic transmission coefficients are lacking. We plan to merge this fission crosssection modeling with modern nuclear reaction codes being developed at LANL and at the CEA. We also plan to use a consistent set of fission barrier parameters derived from macroscopic-microscopic calculations by Möller et al. [3]. The result of a five-dimensional macroscopic-microscopic calculation of potential energy surface is shown in Fig. 3 in the case of ${ }^{240} \mathrm{Pu}$ fissioning, along the quadrupole moment $Q_{2}$. Such calculations can also be used to calculate neutron and proton single-particle states at every point along the deformation coordinate. Along with an understanding of the inertia parameter variation with deformation, one can obtain

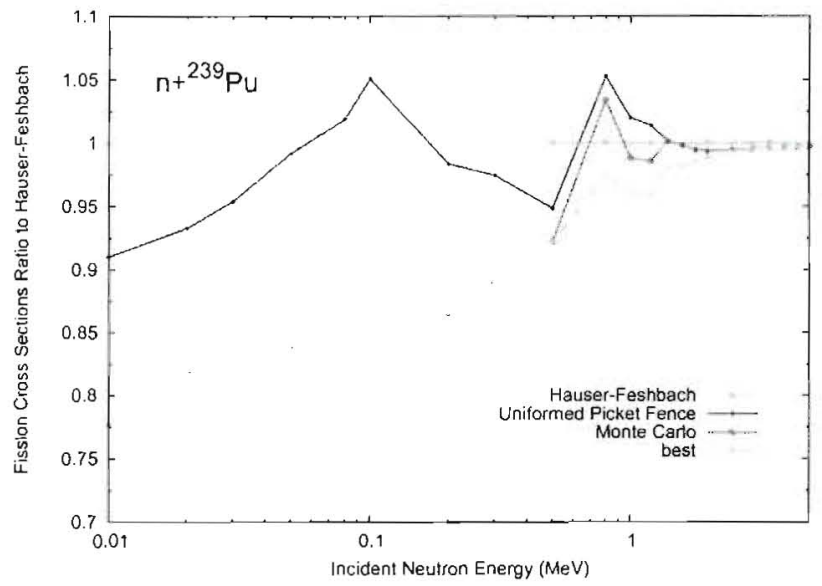

Fig. 2. Neutron-induced fission cross sections of Pu239 calculated under different assumptions: (1) Hauser-Feshbach; (2) uniform picket-fence model; (3) full Monte Carlo.

quasi-particle states and full level densities at the barrier deformations, using a traditional combinatorial approach.

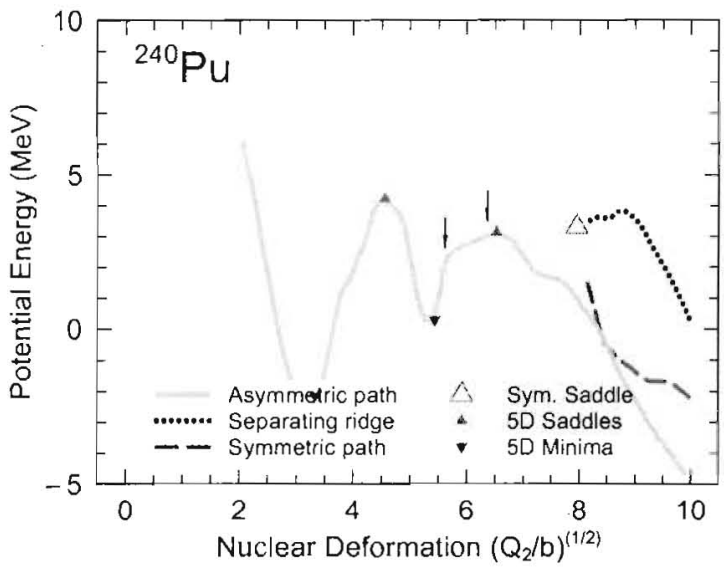

Fig. 3. Fission path of the ${ }^{240} \mathrm{Pu}$ fissioning nucleus, using a macroscopic-microscopic approach [3].

\section{PROMPT FISSION NEUTRONS AND GAMMA RAYS}

Right after the point of scission, two (or more) fragments are formed and move away from each other by Coulomb repulsion. They are usually deformed and excited, and will quickly emit so-called prompt neutrons and gamma rays to reach a more stable configuration. Once they reach their ground-state or an isomeric state, they may further $\beta$ decay, and eventually emit so-called $\beta$-delayed neutrons and 
$\gamma$-rays. In this work, we are only interested in the former, prompt particles.

All modern evaluations of prompt fission neutrons rely on the original or a modified version of the Los Alamos or Madland-Nix model [6]. With only a handful of input parameters, this model has been very successful in computing the average prompt fission neutron spectrum (PFNS) as a function of the incident neutron energy and of the fissioning system. The neutrons are evaporated in Weisskopf-type spectra whose temperatures follow a triangular distribution that corresponds to a specific excitation energy distribution. This calculation is usually performed for only a few fragments centered around the peaks of the fission fragment distribution. Moreover, the neutrons are not emitted sequentially, but rather from an effective spectrum and effective temperature distribution.

Although the original model has been modified in several ways (many fragmentations [7], different temperatures in the light and heavy fragments [8]), it remains limited to the prediction of average quantities, and cannot make predictions of prompt $\gamma$-ray spectrum and multiplicity. To bypass this limitation, we have implemented Monte Carlo simulations of the Hauser-Feshbach equations applied to the de-excitation of the fission fragments.

\subsection{Monte Carlo Hauser-Feshbach Modeling}

In this approach, a primary fission fragment $(A, Z)$ formed with an initial excitation energy $U_{0}$ decays by emitting neutrons, until it becomes energetically impossible. Neutrons are evaporated in a Weisskopf-type spectrum at temperature $T$

$$
\phi(\epsilon) \propto \epsilon \sigma(\epsilon) \exp (-\epsilon / T),
$$

where $\epsilon$ is the center-of-mass neutron energy and $\sigma(\epsilon)$ is the inverse compound nucleus formation cross section. The Monte Carlo technique is used to sample this spectrum, a neutron at a specific energy $\epsilon_{0}$ being chosen, and a new, residual nucleus $(A-1, Z)$ being formed at a residual excitation energy

$$
U_{1}=U_{0}-\epsilon_{0}-B_{n}(A, Z),
$$

with $B_{n}(A, Z)$ the neutron binding energy of the nucleus $(A, Z)$. The assumption of isotropic neutron emission in the center-of-mass reference frame is used to obtain the corresponding neutron energy in the laboratory frame. The sequence of successive neutron emissions is followed until the residual excitation energy is too low, and only $\gamma$ rays can be further emitted.

The primary fission fragment yields $Y(A, Z, T K E)$ as a function of the mass $A$, charge $Z$ and total kinetic energy $T K E$ are reconstructed from experimental data. For a particular pair of fragments and $T K E$ value, the total excitation energy shared by the two fragments is simply given by

$$
U=E_{r}-T K E,
$$

where $E_{r}$ stands for the fission energy release, and simply depends on nuclear masses, taken from the Audi-Wapstra tables [9], on the incident neutron energy and on the neutron binding energy for the target nucleus.

While the total excitation energy $U$ is well characterized for a given $\left(A_{l}, Z_{l}, A_{h}, T_{h}, T K E\right)$ fragment configuration, its distribution among the light and heavy fragments is not known. In our calculations, we introduce the parameter

$$
R_{T}=T_{1} / T_{h}
$$

which represents the ratio of the average temperatures in the light and heavy fragments respectively. It is important to note that these temperatures are not to be considered at the time of scission, but rather at the time of neutron emission, i.e., once both fragments are fully accelerated and with their deformation energies already transformed into intrinsic excitation energies.

Another important ingredient entering in these calculations are the average level density parameters $a(A, Z)$. They are used to go back and forth between excitation energy and nuclear temperature in the Fermi-gas approximation

$$
U=a T^{2}
$$

The Gilbert-Cameron-Ignatyuk formalism is used, and the $a$-parameter has the following energy-dependency

$$
a(A, U)=a^{*}(A)\left\{1+\frac{\delta E}{U}[1-\exp (-\gamma U)]\right\},
$$

where $\delta E$ is the shell correction energy, and $\gamma$ is a damping parameter introduced to account for the washing-out of shell effects with increasing excitation energy. In this work, systematics for the level density parameters were taken from Kawano et al. [10].

\subsection{Numerical Results}

Numerical results are shown here for the low-energy neutron-induced fission of ${ }^{235} \mathrm{U}$. In this case, the experimental fission fragment yields from F.J.-Hambsch [12] were used as a starting point in our Monte Carlo simulations. Note that what was measured are the fission products, i.e., after prompt neutron emission, and an iterative procedure was used to infer the pre-neutron emission fission fragments. Independent direct measurements of the fission fragments are encouraged so that any ambiguity resulting from this iterative procedure gets lifted.

The calculated average prompt neutron multiplicity as a function of the fragment mass is shown in Fig. 4, and compared with experimental data from Nishio [11]. The impact of the $R_{T}$ parameter on $\bar{v}(A)$ is evident from this figure, and tends to shift upwards (resp. downwards) the light fragments curve (resp. the heavy fragments curve) with increasing $R_{T}$ values. This shift is almost constant across the mass range for a given value of $R_{T}$. This result is remarkable as one would expect $R_{r}$ to depend significantly on the specific fragmentation considered. As the energy of the incident neutron is increased, and the intrinsic part of the total excitation energy increases, $R_{T}$ would tend to go toward unity reflecting a fissioning system in thermal equilibrium. 


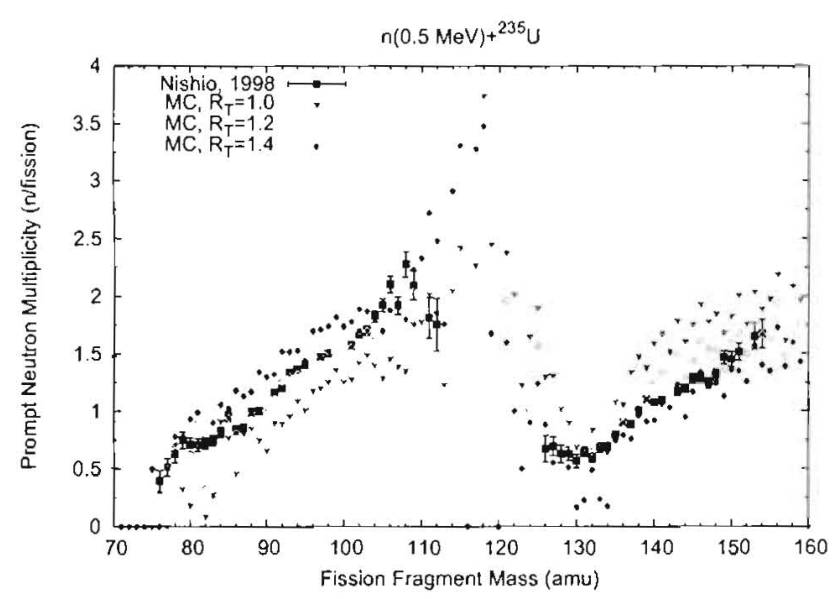

Fig. 4. Average prompt fission neutron multiplicity as a function of fragment mass. The experimental data points are from Nishio et al. [11], and were obtained with thermal neutrons.

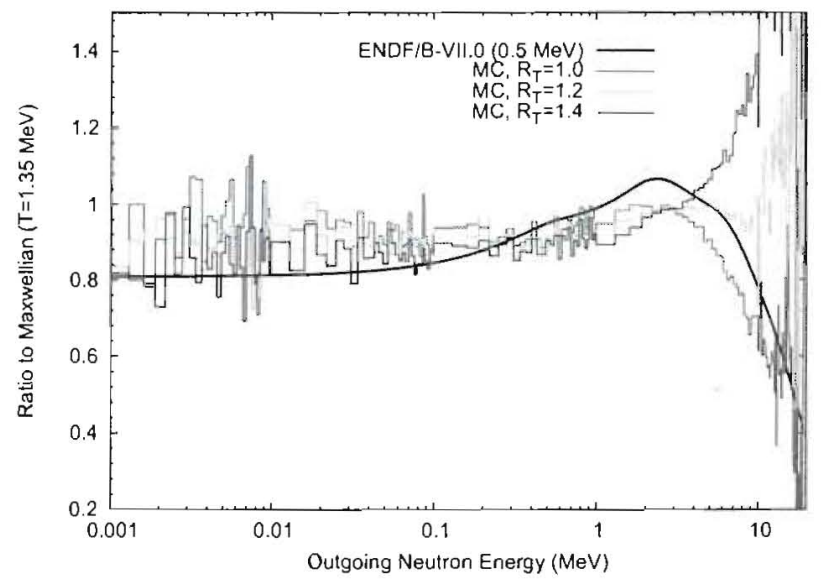

Fig. 5. Prompt fission neutron spectrum calculated in the laboratory frame in the reaction $n(0.5 \mathrm{MeV})+{ }^{235} \mathrm{U}$. The sensitivity of the results to the choice of the $R_{T}$ parameter is emphasized.

The total neutron spectrum calculated in the laboratory frame is shown in Fig. 5, as a ratio to a Maxwellian at temperature $1.35 \mathrm{MeV}$, for different values of the $R_{T}$ parameter. It is also compared to the ENDF/B-VIJ.0 evaluation obtained by applying the Los Alamos model, with parameters fitted to reproduce existing experimental data. Increasing $R_{T}$ values lead to harder spectra, as more neutrons are emitted from the light fragment, for which the intrinsic temperatures are higher than for the heavy fragment. The kinematic boost is also more important for the light than for the heavy fragment, adding to the hardness of the spectrum. On this figure, all calculated spectra are normalized to unity. However, to compare with experimental data, those need to be re-normalized within the energy limits of observation. Figure 6 shows calculated spectra, ENDF/B-VII.0 and Monte Carlo with $R_{T}=1.0$, along with several experimental data sets obtained for thermal as well as $0.5 \mathrm{MeV}$ neutrons. In this case, the Monte Carlo calculations were performed for thermal neutrons. The Monte Carlo results were scaled to provide the same normalization as the experimental data sets in the observed energy range. The results are in very good agreement with the experimental data over the whole energy range. Interestingly, the low-energy part of the calculated spectra, below $100 \mathrm{keV}$, is $10-15 \%$ higher than the ENDF/B-VII.0 result, in agreement with results by Starostov et al. [13]. As some authors have suggested different explanations for this higher spectrum, including the presence of scission neutrons, the present calculations suggest that there is no need for an extra neutron source, but instead that a more exact treatment of the fission fragment decay can account for this.

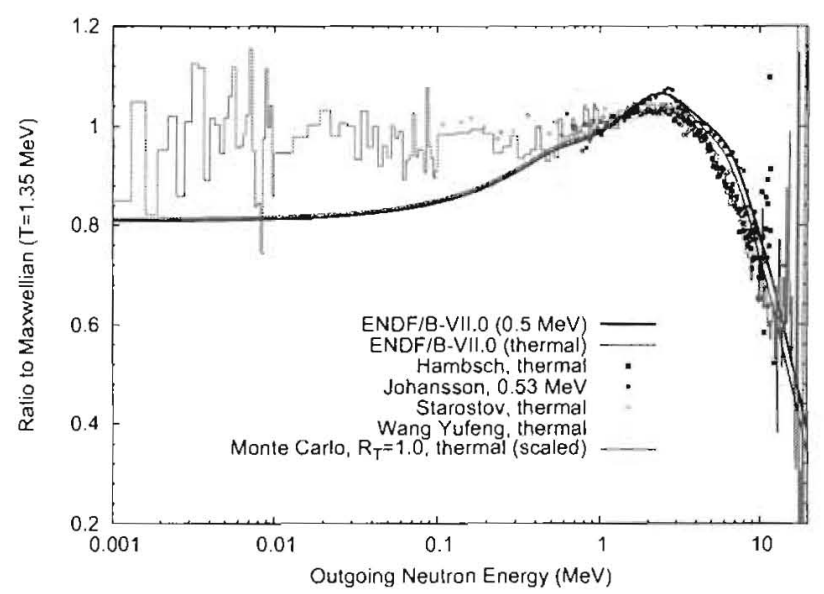

Fig. 6. Same as Fig. 5 but for thermal neutrons. The calculated Monte Carlo result was re-normalized to the experimental data sets on the experimental outgoing energy range. Experimental error bars were removed for sake of clarity.

The calculated exclusive spectra for specific neutron multiplicities $v_{i}$ are shown in Fig. 7. The hardness of the spectrum shows a strong sensitivity to the neutron multiplicity, the spectrum for only I neutron out is significantly harder than the spectrum calculated for 3 neutrons out. This result can be used to devise better strategies for detector efficiency calculations, which always assume that the neutron spectrum is independent on the number of neutrons detected. Advanced Monte Carlo transport calculations could be performed making use of the new data generated by this Monte Carlo approach.

Another quantity of interest is the neutron multiplicity distribution, $\mathrm{P}(v)$. It is shown in Fig. 8, and compared with experimental data by Franklyn [14], Boldeman [15] and Diven [16]. Except for Franklyn's data, which are not direct measurements of $\mathrm{P}(v)$ but instead the result of simulations, the agreement between the Monte Carlo results and the experimental data is remarkable. It also depends only very weakly on the $R_{T}$ value, as the total neutron multiplicity 


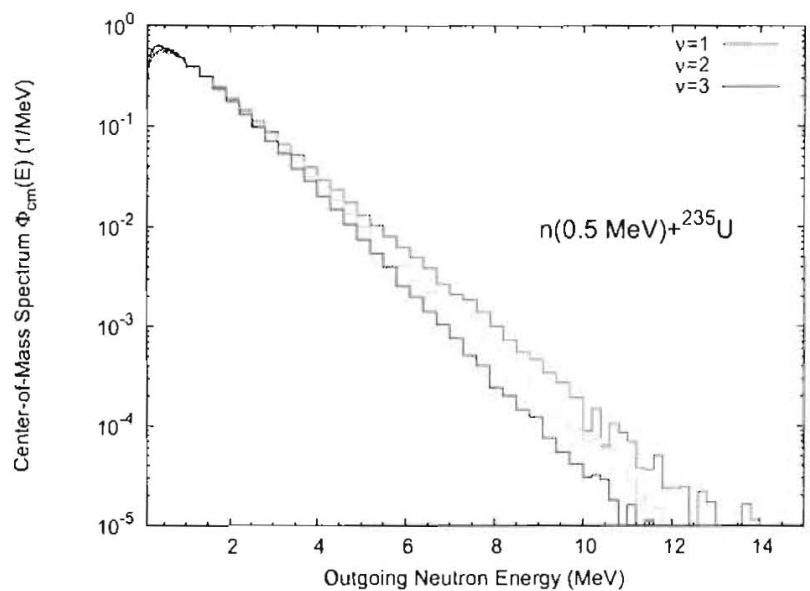

Fig. 7. Exclusive center-of-mass spectra calculated for specific values of the neutron multiplicity $v$.

depends mostly on TXE and very little on how this energy is shared among the primary fragments. Calculated exclusive data such as the ones presented in Figs. 7 and 8 are new results that can/should be incorporated in future evaluated data files, and used in advanced transport simulations.

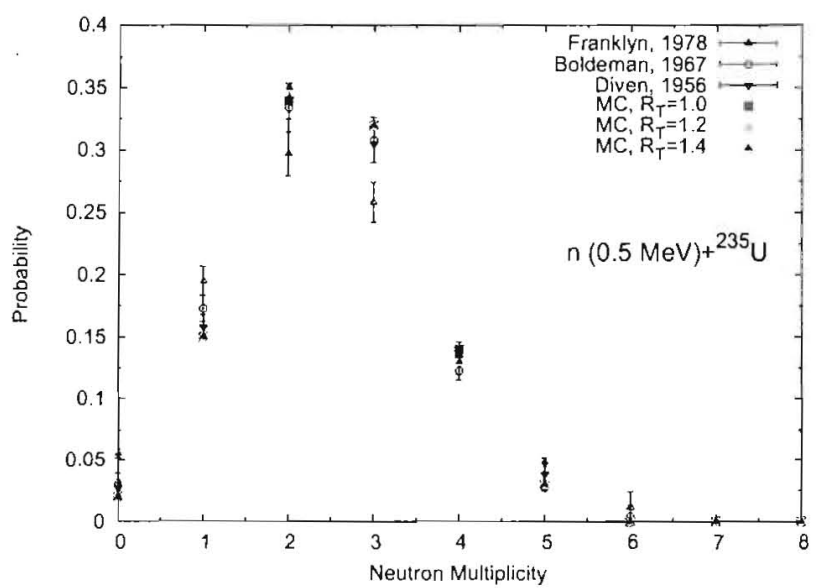

Fig. 8. Prompt neutron multiplicity distribution $\mathrm{P}(v)$ for $\mathrm{n}(0.5$ $\mathrm{MeV})+{ }^{235} \mathrm{U}$.

While those results are very encouraging, the sensitivity of the results to the model parameters has not been tested thoroughly yet. In addition, several approximations have not been lifted yet. For instance, the neutron- $\gamma$-ray competition was not taken into account properly in the present calculations, as $\gamma$-rays can be emitted long before the excitation energy reaches the neutron separation energy if the spin of the fragments is considered, i.e., if the Yrast line is reached. Very little is known on the initial spin distribution of the fragments, and one has to rely on adjusted parameters to perform such calculations. Other parameters, such as the level density parameters and deformation energies of the initial fragments, should be well constrained before extending this approach 10 more isolopes. This work is underway.

\section{THE NEXT GENERATION OF EVALUATION TOOLS FOR NUCLEAR FISSION}

At this stage, an all-encompassing evaluation tool for fission data remains elusive. While not tackling the full complexity of the fission problem, the two examples above, i.e., advanced modeling of fission cross sections and prompt fission neutrons and $\gamma$ rays, show that it is indeed possible to move in that direction. In particular, it is important for any future evaluation work on fission to be performed with a global view in mind. Fission fragment distributions should be linked to fission cross sections, as well as prompt fission neutrons and gamma rays. It is also important to perform such calculations not only for a select few, but for a large set of isotopes, linked either through experimental setups (e.g., fission cross section ratio measurements) or through physical constraints (e.g., multi-chance fissions in a suite of isotopes). Such a global approach will lead to less phenomenology, more physical constraints on the remaining model parameters, and more predictive capabilities.

\section{ACKNOWLEDGMENTS}

\section{REFERENCES}

[1] N. Bohr and J.A. Wheeler, Phys. Rev. 56, 426 (1939).

[2] S. Goriely, S. Hilaire, A.J. Koning, M. Sin, R. Capote, Phys. Rev. C79, 024612 (2009).

[3] P. Möller, A.J. Sierk, T. Ichikawa, A. Iwamoto, R. Bengtsson, H. Uhrenholt, S. Åberg. Phys. Rev. C79, 064304 (2009).

[4] S. Bjørnholm, J.E. Lynn, Rev. Mod. Phys. 52, 725 (1980).

[5] J.E. Lynn and A.C. Hayes, Proc. Third Int. Conf. on Fission and Properties of Neutron-Rich Nuclei, Sanibel Island, Florida (2002).

[6] D.G. Madland, J.R. Nix, Nucl. Sci. Eng. 81, 213 (1982).

[7] G. Vladuca, A. Tudora, Ann. Nucl, Energy 28, 419 (2001).

[8] T. Ohsawa, INDC (NDS) report 251 (1991).

[9] G. Audi, A.H. Wapstra, C. Thibault, Nucl. Phys. A729, 337 (2003).

[10] T. Kawano, S. Chiba, H. Koura, J. Nucl. Sci. Tech. 43, I (2006).

[11] K. Nishio, Y. Nakagome, H. Yamamoto, I. Kimura, Nucl. Phys. A632, 540 (1998).

[12] F.-J. Hambsch, private communication.

[13] V.N. Nefedov, B.I. Starostov, and A.A. Boytsov, Sixth AllUnion Conference on Neutron Physics, Kiev, p.285 (1983).

[14] C.B. Franklyn, C. Hofmeyer, and D.W. Mingay, Phys. Lett. 78B, 564 (1978).

[15] J.W. Boldeman and A.W. Dalton, AAEC/E172, Australian Atomic Energy Commission, Lucas Heights (1967).

[16] B.C. Diven, H.C. Martin, R.F. Taschek, and J. Terrell, Phys. Rev. 101, 1012(1956). 